\title{
Educación y desigualdad del ingreso en municipios del estado de Chihuahua
}

\author{
Javier Martínez Morales \\ Jesús Hernández Arce \\ Herik Germán Valles Baca
}

Profesores investigadores Universidad Autónoma de Chihuahua

\section{Resumen}

1 siguiente trabajo caracteriza la es$\checkmark$ colaridad a través de los años pro$\checkmark$ medio del trayecto formativo y el coeficiente de Gini de Escolaridad, y estima el efecto de ésta sobre la desigualdad del ingreso. Al mismo tiempo se calculó el coeficiente de Gini, por medio de la variable de ingresos por trabajo mensuales. Para el análisis del efecto de la escolaridad sobre la distribución del ingreso se consideró la base de microdatos del Censo de Población y Vivienda 2010. Los resultados muestran que la escolaridad no tiene un efecto significativo sobre la disminución de la desigualdad del ingreso.

Palabras clave: educación, ingresos, promedio de escolaridad, desigualdad.

\section{Introducción}

La desigualdad del ingreso en México ha sido un tema que genera una controversia en cualquier hacedor de política pública. En la gran mayoría de los casos el argumento hace hincapié en que son diferentes factores internos y externos lo que hacen que la desigualdad del ingreso se polarice.

Para agravar la situación, México tiene un total de diez de los mil 645 millonarios que contempla la revista Forbes. Para pertenecer a la lista se debe contar con una fortuna mínima de mil millones de dólares, Carlos Slim llega a los 72 mil millones de dólares, lo que lo posiciona en el segundo lugar de las personas más ricas del mundo (Forbes, 2014). 
Un indicador para medir la desigualdad del ingreso es a través del coeficiente de Gini. De acuerdo con los datos de la Secretaria de Desarrollo Social (2013), calculados por el Consejo Nacional de Evaluación de la Política de Desarrollo Social, en 1992 el coeficiente de Gini alcanzó un valor de 0.543 mientras que en el año 2012 fue de 0.498 , esto significó una reducción de apenas el $0.43 \%$ del promedio anual, mientras que durante todo el periodo la reducción fue de $8.20 \%$. Dado lo anterior, ¿qué efecto, si es que lo ha tenido, ha jugado la escolaridad en la pequeña reducción en la desigualdad de los ingresos?

La escolaridad ha sido considerada como un catalizador para compensar la alta desigualdad de ingreso en el país. De ahí que las investigaciones busquen estimar el efecto de la escolaridad sobre la desigualdad del ingreso. Por tal motivo esta investigación tiene como objetivo principal caracterizar la escolaridad a través de dos indicadores: años promedio de escolaridad y coeficiente de Gini de educación; y medir el efecto que tiene sobre la desigualdad del ingreso para los 67 municipios del estado de Chihuahua. La hipótesis que se plantea recae en el hecho que la escolaridad no tiene un efecto compensatorio sobre la desigualdad del ingreso.

\section{Años promedio de escolaridad, desigualdad educativa y desigualdad del ingreso}

El trabajo de Bracho (1994) muestra la relación que existe entre la desigualdad educativa, medida por desviación estándar y los años promedio de escolaridad; los resultados revelan que la correlación entre estas dos variables no tienen una tendencia lineal sino cuadrática en forma de u invertida, otros estudios confirman esta relación (Ram, 1990; De Gregorio y Lee, 1999; Thomas, Wang y Fan, 2000).

Martínez y Fernández (2013) desarrollan cuatro indicadores de desigualdad educativa para los municipios del estado de Chihuahua: años promedio de escolaridad, índice de Gini, desviación estándar y curva de Lorenz; en el periodo 1960-2010, los resultados encontrados evidencian que a mayor promedio de escolaridad menor Gini de educación. Uno de sus resultados verifica que una mayor desigualdad de la escolaridad conlleva a una mayor desigualdad del ingreso.

De Gregorio y Lee (1999) investigan la relación que guarda la desigualdad del ingreso y la escolaridad. Consideran a un grupo de 106 países dentro del periodo de 1960 al 2000. Sus resultados confirman que un incremento de la educación no produce un efecto significativo en la reducción de la desigualdad del ingreso y esto recae al incremento de la desigualdad educativa.

Martínez Ruíz (2000) argumenta que la desigualdad educativa, medida por el coeficiente de Gini, disminuyó de 1970 al 2000 para las entidades federativas; encontró que existe una relación negativa casi perfecta entre desigualdad educativa y años promedio de escolaridad. La investigación muestra que al compararse la desigualdad del ingreso contra la desigualdad de la educación, los resultados pueden contrastarse llegando al hecho de que esta desigualdad 
ha ido disminuyendo a través del tiempo mientras que el ingreso puede aumentar o disminuir.

Barceinas (2004) afirma que la escolaridad reduce la desigualdad del ingreso, en cambio deja abierto al debate si este efecto puede deberse a causas exógenas, por ejemplo al crecimiento económico de un país. Finalmente concluye que el mejor efecto para aminorar la concentración del ingreso es la distribución de la educación.

Un estudio realizado en 2010 determinó que la escolaridad no llega a ser un ecualizador de los ingresos, que las causas principales son una desigualdad inicial en la distribución de los recursos económicos y la dotación de factores de la sociedad (Figueroa, 2010).

\section{Estrategia metodológica}

Para el análisis del efecto de la escolaridad sobre la distribución del ingreso, de los municipios del Estado de Chihuahua, se consideró la base de microdatos del Censo Poblacional 2010 del Instituto Nacional de Estadística, Geografía e Informática (INEGI). La decisión de considerar esta base fue porque contiene información a nivel persona y por municipio y se pueden generar indicadores como el índice de Gini y obtener el ingreso promedio de los municipios. La base final se conformó de 73024 observaciones para los 67 municipios del estado, con edad mayor o igual a 15 años e ingresos superiores o iguales a los mil 500 pesos mensuales.

Para la generación de indicadores de desigualdad educativa se utilizó la meto- dología de Thomas, Wang y Fan (2000). El coeficiente de Gini de educación se calcula siguiendo la siguiente fórmula:

$$
E_{L}=\left(\frac{1}{\mu}\right) \sum_{i=2}^{n} \sum_{j=1}^{i-1} p_{i}\left|y_{i}-y_{j}\right| p_{j}
$$

Donde:

$\mathrm{E}_{\mathrm{L}}=$ es el coeficiente de Gini de educación

$\mu=$ los años promedio de escolaridad

$\mathrm{p}_{\mathrm{i}}$ y $\mathrm{p}_{\mathrm{j}}$ son los porcentajes de la población con cierto grado de escolaridad

$\mathrm{y}_{\mathrm{i}}$ e $\mathrm{y}_{\mathrm{j}}$ son los años de escolaridad que se alcanzan en los diferentes niveles de educación.

$\mathrm{N}$ es el número de categorías que existen de acuerdo con los datos, para este caso $\mathrm{n}=9$, es decir, sin escolaridad, primaria incompleta, primaria completa, secundaria incompleta, secundaria completa, bachillerato incompleto, bachillerato completo, universidad completa y posgrado. El coeficiente de Gini de educación toma valores entre cero y uno, cero es una equidad perfecta mientras que 1 es una desigualdad perfecta. De aquí en adelante, este coeficiente se considerará como desigualdad educativa.

El cálculo del coeficiente de Gini, que mide la desigualdad del ingreso, y el nivel de deciles se elabora a través del programa Distributive Analysis Stata Package (DASP) que se instala dentro del paquete Stata 11. Para la interpretación del coeficiente de Gini se aplica la misma regla que el coeficiente de Gini de educación. 
REVISTA DE INVESTIGACIÓN EDUCATIVA DE LA REDIECH N. 9

ISSN: 2007-4336

\section{Resultados}

Del total de los datos $29.45 \%$ fueron mujeres y $70.55 \%$ fueron hombres. La edad promedio de los hombres fue de 38 años, mientras que la de las mujeres alcanzó los 36 años.
En la figura 1 se observa que una mayor escolaridad para el hombre implica un nivel de ingreso promedio más elevado, sin embargo la mujer, a pesar de tener un mismo grado de escolaridad, no ve reflejado este mismo nivel de ingreso.

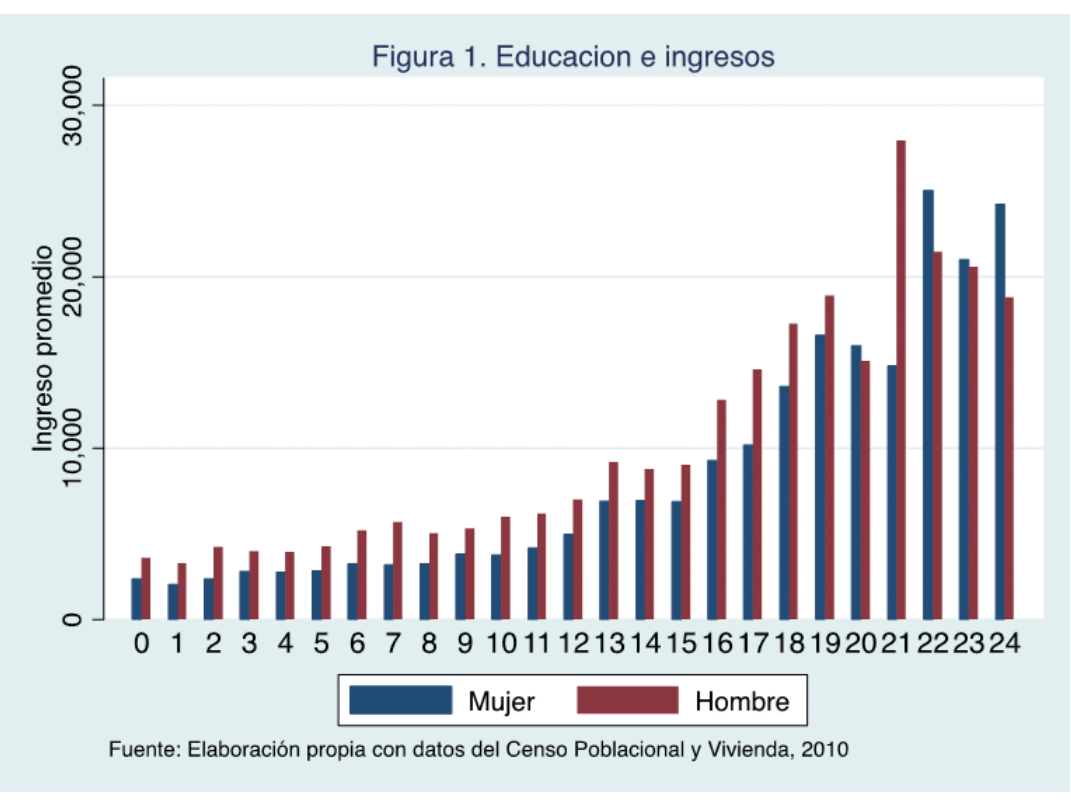

La tabla 1 muestra datos tanto de ingreso como de escolaridad por deciles, el cociente entre el decil X y I es de 10.43, es decir; el decil más rico concentra diez veces más ingreso que el decil más pobre. La desigualdad de la escolaridad disminuye en los deciles más ricos, los años promedios de escolaridad para el decil I representan 1 de secundaria mientras que para el decil $\mathrm{X}$ alcanzan 1 año de universidad. Finalmente una persona que pertenece al decil I gana apenas 1,965.69 pesos mensuales, mientras que una del decil $X$ gana 22,401.38 pesos mensuales, que equivale a ganar 11 veces más en el decil más rico que en el más pobre.

\begin{tabular}{|c|c|c|c|c|}
\hline Municipio & $\begin{array}{l}\text { Ingreso } \\
\text { promedio }\end{array}$ & $\begin{array}{l}\text { Gini de } \\
\text { ingreso }\end{array}$ & APE & $\begin{array}{c}\text { Gini de } \\
\text { educación }\end{array}$ \\
\hline Allende & 4751.38 & 0.431 & 8.83 & 0.31 \\
\hline Hidalgo del Parral & 5574.22 & 0.433 & 9.63 & 0.27 \\
\hline Jiménez & 5068.33 & 0.424 & 9.56 & 0.26 \\
\hline López & 4544.16 & 0.409 & 8.66 & 0.28 \\
\hline Matamoros & 4402.95 & 0.343 & 8.43 & 0.25 \\
\hline San Francisco del Oro & 5918.95 & 0.390 & 9.64 & 0.27 \\
\hline Santa Bárbara & 5247.96 & 0.354 & 9.42 & 0.25 \\
\hline Valle de Zaragoza & 5225.32 & 0.470 & 7.40 & 0.30 \\
\hline Estatal & 5658.77 & 0.437 & 8.85 & 0.28 \\
\hline
\end{tabular}


La tabla 2 representa solo indicadores muestran que un alto grado de escolaridad de ingreso y escolaridad para la región sur no se refleja en una menor desigualdad de del estado de Chihuahua, los resultados ingresos.

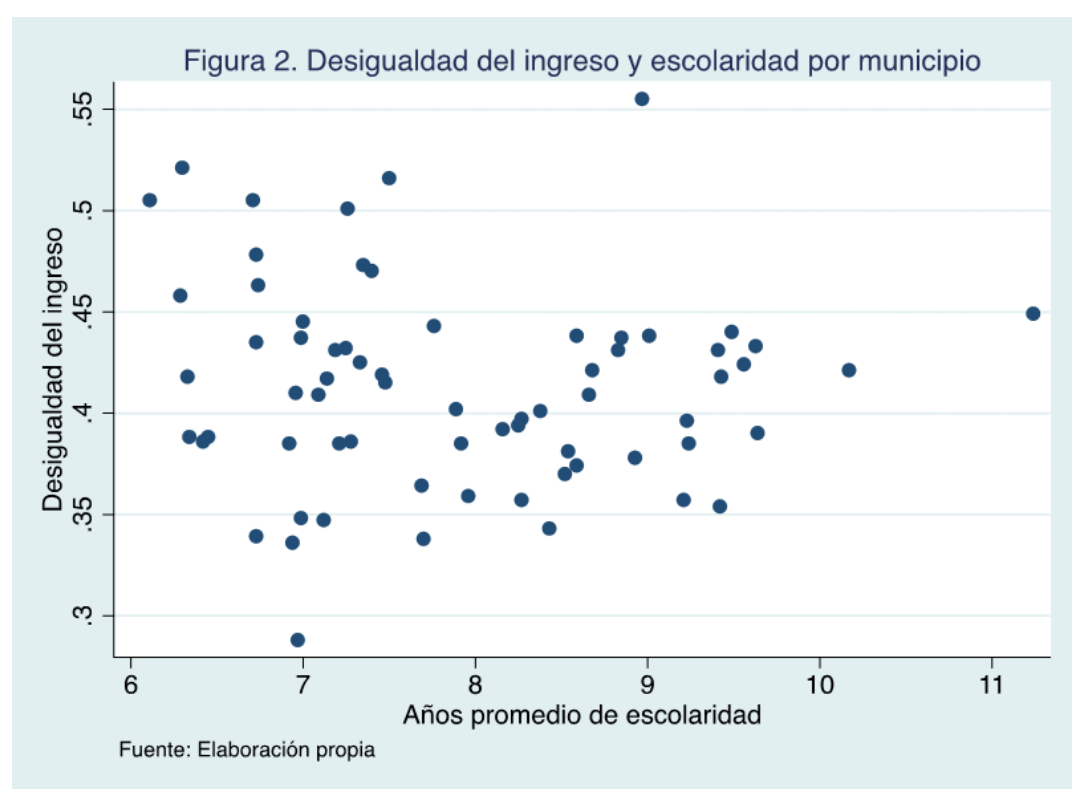

La figura 2 incorpora el total de observaciones de los 67 municipios del estado, lo que confirma los datos de la tabla 2 que no hay una clara relación entre la desigualdad del ingreso y escolaridad.

De acuerdo con la tabla 3 la correlación entre Gini de ingreso y años promedio de escolaridad es negativa pero cercana a cero, mientras que la correlación entre desigualdad del ingreso y desigualdad educativa es positiva pero baja. En el caso de años promedio de escolaridad y Gini de educación hay una relación positiva y alta y lo mismo sucede con ingresos promedio y años de escolaridad.

Cuadro 3. Correlación entre variables de estudio

\begin{tabular}{|c|c|c|c|c|}
\hline & $\begin{array}{l}\text { Gini de } \\
\text { ingreso }\end{array}$ & $\begin{array}{c}\text { Gini de } \\
\text { educación }\end{array}$ & $\begin{array}{l}\text { Años promedio } \\
\text { de escolaridad }\end{array}$ & $\begin{array}{l}\text { Ingreso } \\
\text { promedio }\end{array}$ \\
\hline Gini de ingreso & 1 & & & \\
\hline Gini de educación & 0.1175 & 1 & & \\
\hline $\begin{array}{l}\text { Años promedio de } \\
\text { escolaridad }\end{array}$ & -0.0775 & -0.4501 & 1 & \\
\hline Ingreso promedio & 0.256 & -0.5583 & 0.3589 & 1 \\
\hline
\end{tabular}

Para llevar a cabo un análisis estadístico más riguroso se presenta el siguiente modelo econométrico:

$$
\text { Gini }_{i}=\alpha+\beta_{1} A P E_{i}+\beta_{2} \text { Ginieduc }_{i}+\beta_{3} \text { ling }_{i}+\mu_{i}
$$


REVISTA DE INVESTIGACIÓN EDUCATIVA DE LA REDIECH N. 9

ISSN: 2007-4336

Cuadro 4. Efecto de la Escolaridad sobre el ingreso

\begin{tabular}{|c|c|c|c|c|}
\hline Observaciones & 68 & & & \\
\hline Prueba F & 0.0053 & & & \\
\hline R2 & 0.1321 & & & \\
\hline \multirow[t]{2}{*}{ Variable dependiente } & \multicolumn{2}{|c|}{ Gini de ingreso } & & \\
\hline & Coeficiente & $\begin{array}{c}\text { Error } \\
\text { estándar }\end{array}$ & $\mathrm{t}$ & $P>t$ \\
\hline Gini de Educacion & 0.834379 & 0.299132 & 2.79 & 0.007 \\
\hline $\begin{array}{l}\text { Años promedio de } \\
\text { Escolaridad }\end{array}$ & -0.00941 & 0.01322 & -0.71 & 0.479 \\
\hline Logaritmo del ingreso & 0.252367 & 0.098453 & 2.56 & 0.013 \\
\hline Constante & -3.21164 & 0.887305 & -3.62 & 0.001 \\
\hline
\end{tabular}

Donde:

Gini es el índice que mide la desigualdad de ingresos

APE representa los años promedio de escolaridad

Ginieduc es el índice de Gini de educación que mide la desigualdad de la escolaridad

Ling es el logaritmo del ingreso promedio de los individuos que viven en el municipio i.

Los resultados obtenidos de la regresión se reflejan en la tabla 4 .

El Gini de educación resultó ser significativo y positivo, manteniendo las demás variables constantes un incremento de un punto de la desigualdad educativa, la desigualdad del ingreso se incrementa en 0.83 puntos. Mientras que los años promedio de escolaridad resultaron negativos pero no significativos. Por último, el logaritmo del ingreso resulto ser significativo y positivo, es decir; un incremento de 1\% del ingreso reduce la desigualdad educativa en 0.0025 puntos, manteniendo todo lo demás constante.

\section{Conclusiones}

Una de las limitantes del estudio recae en el hecho de que solo tenemos datos del Censo de Población y Vivienda 2010, por lo cual no permite hacer una análisis panel por más años.

El generar indicadores de educación permitió mostrar que mayores años promedio de escolaridad reducen la brecha en la desigualdad educativa, medida a través del coeficiente de Gini. Al mismo tiempo mayores grados de escolaridad generan un mejor nivel de ingreso, aunque este mejor nivel de ingreso no implique una reducción de la desigualdad del mismo, esto puede deberse al hecho de que altos grados de escolaridad solo beneficien a los deciles más altos de la distribución del ingreso.

Finalmente, de acuerdo con la evidencia econométrica, la escolaridad no tiene un efecto significativo en la reducción de la desigualdad del ingreso a pesar de que la desigualdad educativa se está reduciendo; sin embargo a nivel deciles, sigue habiendo una amplia brecha de desigualdad. Esto último hace pensar que existen factores 
externos que hacen que la escolaridad no tenga un efecto compensatorio en la disminución de la desigualdad del ingreso.

\section{Referencias}

Barceinas, F. (2004). Educación y distribución del ingreso en México. Siteal. Buenos Aires.

Bracho, T. (1994). Distribución y desigualdad educativa en México 1990. Cide. Cuadernos de Trabajo. Estudios Políticos. 16.

De Gregorio, J. y Lee (1999). Education and Income Distribution: New Evidence from Cross-Country Data. Development Discussion Papers. No 714.

Figueroa, A. (2010). Mejora la distribución del ingreso con la escolaridad. El caso de Perú. Revista CEPAL. Vol. 102.
Forbes (2014). Recuperado septiembre 3, 2014 en http://www.forbes.com/sites/luisakroll/2014/03/03/ inside-the-2014-forbes-billionaires-list-facts-andfigures/

Martínez, J. y Fernández, A. (2013). Indicadores de Desigualdad Educativa y sus implicaciones: Caso Chihuahua. Investigación Multidisciplinaria. ITESM. Edición ICM 2013.

Ram, R. (1990). Educational Expansion and Schooling Inequality: International Evidence and Some Implications. The Review of Economics and Statistics. Vol.72. No. 2.

Thomas, V.; Wang y Fan. (2000). Measuring Education Inequality: Gini Coefficients of Education. World Bank Institute.

Secretaría de Desarrollo Social (2013). Indicadores Básicos del Sector de Desarrollo Social. Subsecretaría de Prospectiva, Planeación y Evaluación. Dirección General de Análisis y Prospectiva.

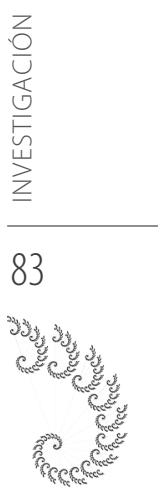

